Document downloaded from:

http://hdl.handle.net/10251/52878

This paper must be cited as:

Camba, J.; Contero, M.; Salvador Herranz, GM. (2014). Implementation challenges of annotated 3D models in collaborative design environments. Lecture Notes in Computer Science. 8683:222-229. doi:10.1007/978-3-319-10831-5_33.

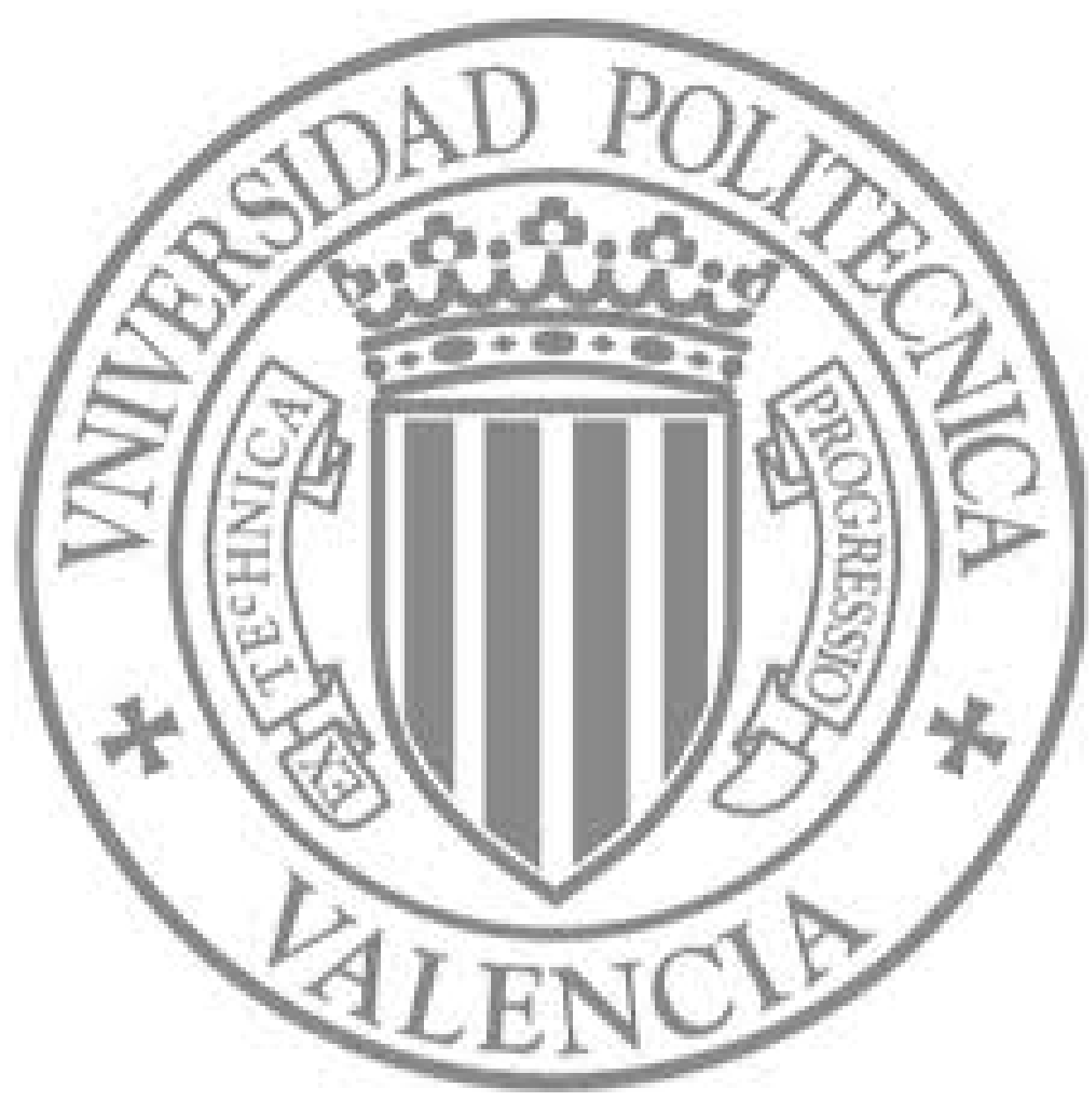

The final publication is available at

http://dx.doi.org/10.1007/978-3-319-10831-5_33

Copyright Springer Verlag (Germany) 


\section{Contribution:}

Camba, J., Contero, M., \& Salvador-Herranz, G. (2014). Implementation Challenges of Annotated 3D Models in Collaborative Design Environments. In Cooperative Design, Visualization, and Engineering (pp. 222-229). Springer International Publishing.

Springer International Publishing AG, Cham (hereinafter called Springer) has the exclusive, sole, permanent, world-wide, transferable, sub-licensable and unlimited right to reproduce, publish, distribute, transmit, make available or otherwise communicate to the public, translate, publicly perform, archive, store, lease or lend and sell the Contribution or parts thereof individually or together with other works in any language, in all revisions and versions (including soft cover, book club and collected editions, anthologies, advance printing, reprints or print to order, microfilm editions, audiograms and videograms), in all forms and media of expression including in electronic form (including offline and online use, push or pull technologies, use in databases and networks for display, print and storing on any and all stationary or portable end-user devices, e.g. text readers, audio, video or interactive devices, and for use in multimedia or interactive versions as well as for the display or transmission of the Contribution or parts thereof in data networks or seach engines), in whole, in part or in abridged form, in each case as now known or developed in the future, including the right to grant further time-limited or permanent rights. For the purposes of use in electronic forms, Springer may adjust the Contribution to the respective form of use and include links or otherwise combine it with other works. For the avoidance of doubt, Springer has the right to permit others to use individual illustrations and may use the Contribution for advertising purposes.

The copyright of the Contribution will be held in the name of Springer. Springer may take, either in its own name or in that of copyright holder, any necessary steps to protect these rights against infringement by third parties. It will have the copyright notice inserted into all editions of the Contribution according to the provisions of the Universal Copyright Convention (UCC) and dutifully take care of all formalities in this connection in the name of the copyright holder.

The final publication is available at Springer via:

http://dx.doi.org/10.1007/978-3-319-10831-5_33 


\title{
Implementation Challenges of Annotated 3D Models in Collaborative Design Environments
}

\author{
Jorge Camba $^{1}$, Manuel Contero ${ }^{2}$, Gustavo Salvador-Herranz ${ }^{3}$ \\ ${ }^{1}$ Engineering Design Graphics, Texas A\&M University, College Station, TX, USA \\ camba@tamu.edu \\ ${ }^{2}$ I3BH, Universitat Politècnica de València, València, Spain \\ mcontero@upv.es \\ ${ }^{3}$ Dpto. Exp. Gráfica, Proyectos y Urbanismo, Univ. CEU Cardenal Herrera, València, Spain \\ gsalva@uch.ceu.es
}

\begin{abstract}
Recent studies in the area of collaborative design have proposed the use of 3D annotations as a tool to make design information explicitly available within the 3D model, so that different stakeholders can share information throughout the product lifecycle. Annotation practices defined by the latest digital definition standards have formalized the presentation of information and facilitated the implementation of annotation tools in CAD systems. In this paper, we review the latest studies in annotation methods and technologies and explore their expected benefits in the context of collaborative design. Next, we analyze the implementation challenges of different annotation approaches, focusing specifically on design intent annotations. An analysis of the literature suggests that the use of annotations has a positive effect on collaborative design communication as long as proper implementation practices, tools, and user interaction mechanisms are in place.
\end{abstract}

Keywords: annotated 3D models, collaborative design, design communication.

\section{Introduction}

Globalization and advances in manufacturing and information technologies are driving engineering organizations towards concurrent distributed design processes, which allow for reduced development times and costs. In this context, communication and coordination are two of the most critical activities for effective teamwork and overall organizational performance [1, 2]. Modern engineering teams, often comprised of specialists from various backgrounds must frequently work together in environments coordinated through technologies such as Product Lifecycle Management (PLM) systems [3]. Communication is critical, as a significant portion of engineers' time is spent exchanging information [4].

In the context of collaborative methodologies, the role of CAD models has been progressively transformed from mere representations of 3D geometry to elements that carry design information and can be shared among designers throughout the different stages of the product lifecycle [5-7]. Some reasons for this change include the 
popularization of the Model-Based Engineering (MBE) paradigm [8] and related technologies. In recent years, an interest in 3D annotation techniques as built-in knowledge repositories has been increasing. The development of standards for Digital Product Definition Data Practices [9, 10] and the implementation of these standards in major $\mathrm{CAD}$ packages have encouraged researchers to explore annotations as mechanisms to explicitly communicate design information. The significance of annotations and their role to mediate interactions were described by many authors [11-13]. A number of prototypes have also been developed [12, 14, 15]. Nonetheless, it is agreed that these roles are poorly addressed by current industrial tools.

In this paper, we review recent advances in annotation technologies and explore its expected benefits in the context of collaborative design. Next, we analyze the challenges of putting different annotation methods into practice, focusing specifically on annotations that communicate design information. Finally, we conclude our paper with a summary and a discussion of future research directions.

\section{Annotations in Computer Aided Design}

In Computer-Aided Design, the term annotation refers to a piece of information (usually text) that points to a specific aspect of the 3D model and provides additional explanations about the part [16]. Because annotations are linked to the geometry of the model, they are also called model-based annotations or $3 \mathrm{D}$ annotations. Annotations have been used to complement engineering drawings by providing information that is difficult or impossible to convey otherwise, such as manufacturing instructions and tolerances. Despite support from CAD packages for many years, most tools were proprietary and software-dependent, which made information exchange difficult. The lack of common rules created inconsistencies, which has had negative impacts in the adoption of annotations in industrial environments.

With the high demands of industry, researchers began to study the suitability of 3D annotations to carry design information $[12,15,17]$. The knowledge captured in CAD models is not merely helpful for design; it represents a major source of value for an organization. This knowledge includes the modeling process and its design intent. Many researchers noted that the efficient communication of design intent has a direct impact on reusability, which is a key issue to leverage current parametric CAD systems $[6,18,19]$. The importance of an explicit representation of design intent was summarized by [18] and yields the following benefits:

- In complex projects, the ability to store, process, and retrieve information about design changes can significantly improve productivity.

- When design intent information is represented explicitly and is easily available for review, the overall quality of the product increases.

- Explicit representation leads to a better use of resources and knowledge.

- Efficient communication of design intent is essential for integrating solutions and transferring design knowledge.

Companies have reported savings by capitalizing on reusable design elements [6] and identified obstacles involved in implementing reusability practices as well as the procedures to mitigate them. One obstacle was stated as "only original designer can 
change models successfully" and the procedure as "detail design information in model." Although standards have formalized how some of this information is presented, to ensure reusability design intent must be added so users understand how and why models were created in a specific manner. Because of its heterogeneous nature, managing design information is a difficult task, and the use of annotations has proven to be promising but challenging. In the next section, we discuss the challenges involved in implementing annotation mechanisms with the purpose of communicating design intent information.

\section{Implementation Challenges}

While specific challenges in using annotated models as carriers of design knowledge have been identified [16, 20], it is useful to review the scientific literature and assess the practical application of the proposed solutions to try to determine the direction of future developments. In addition to the review, we have elaborated on the subject, identified new challenges, and suggested our own approaches. We describe five major challenges related to the practical implementation of annotations: storage, representation, interface, visualization, and user motivation.

\subsection{Annotation Storage}

Annotations require efficient data structures to represent information. These structures demand tools to store, visualize, and interact with the content as well as instruments to manage the anchoring mechanism of the annotation [16]. It is also necessary that representations are unified to make annotations platform-independent and avoid compatibility and portability issues [20].

Based on how data is stored, annotations are classified as in-line (internal), standoff (external), and hybrid. [15, 21]. In-line annotations store the information internally within the model, whereas stand-off annotations save the information in an external repository. The pros and cons of these methods are shown in Table 1. Hybrid approaches combine the strengths of both methods.

Stand-off annotations are generally more appropriate for use with CAD models $[20,22]$, particularly if the data needs to be shared. Since the information is kept separately, they allow flexible updates of the data without affecting the geometry of the model. Additionally, multiple annotation files can be linked to the same model to provide different annotated views to different users [22]. In terms of implementation, eXtensible Markup Language (XML) and SQL databases (particularly, in collaborative environments with PLM systems) have been recognized as common data description standards $[13,23]$. Nevertheless, stand-off annotations are difficult to implement in distributed environments, mainly because of the problem of persistent references [24], which describes the inconsistencies generated in the annotation structures when the geometry of the model being annotated changes or when there is a simultaneous writing access to the model from multiple users. Hybrid representation approaches have been proposed, where annotation information is stored both externally and internally $[15,24]$. 
Table 1. Annotation representation strategies (adapted and extended from [21])

\begin{tabular}{lll}
\hline Strategy & Pros & Cons \\
\hline In-line & Easy implementation & Original document changes \\
& Wide applications & Difficult to have multiple \\
& Full integration with the model (low maintenance) & independent sets of markup \\
& Efficiency in terms of processing and manipulation & Difficult to share information \\
& Already supported by most CAD systems & in collaborative environments \\
Stand-off & Non-change of representation for the original object & Difficult to implement \\
& $\begin{array}{l}\text { Support of multiple independent sets of markup } \\
\text { Support of progressively information update }\end{array}$ & Persistent references \\
& $\begin{array}{l}\text { Leorganization of information for different } \\
\text { purposes and applications }\end{array}$ & method of references \\
& Easy distribution of information. & File maintenance \\
& Information can be processed separately. & \\
\hline
\end{tabular}

\subsection{Annotation Representation}

An additional challenge regarding the implementation of annotated models involves the annotation content structure, i.e. what information needs to be included and in what form, so information is communicated effectively. Naturally, decisions need to be made as to how design intent can be captured and communicated using annotations. Although some semi-automated capturing tools have been implemented $[11,25,26]$ (many of them based on IBIS [27]), capturing design intent is a task that cannot be completely automated [28], requiring designers to be properly trained.

To provide computational support, design intent information must be represented in a structured manner [28]. With a formal syntax, it is relatively simple for a computer to process this information. However, fixed structures can also limit expressiveness and become intrusive to the user, which has in fact hindered the adoption of these tools in industry. For a designer, it is more intuitive to use natural language, particularly because of the difficulty of representing heterogeneous information (such as design intent) with fixed structures. A recent approach proposed by [29] suggests logging the actions performed by a designer in a CAD session and interpreting patterns found in these actions, which minimizes user intervention in the process. Regardless of the technology, when users are allowed to use natural language, new challenges appear, such as minimizing the effects of writing style and language on communication effectiveness, determining the optimum annotation length so annotations are not ignored, and implementing natural language processing mechanisms so computational support can still be provided.

\subsection{Annotation Interface}

Methods to support interaction with annotations must allow users to enter and retrieve data easily and intuitively [16], as designers are often reluctant to spend additional time adding information to their models [30]. The lack of adequate tools for knowledge-acquisition is in fact the major cause for the knowledge-acquisition 
bottleneck [30]. Interface simplicity and integration with existing tools are crucial factors for the successful implementation of design annotations.

Although a number of prototypes have been developed [12, 14, 15], integration of the annotation tools with the CAD application provides users with an already familiar interface, which minimizes the learning curve and the need to constantly switch between applications. In this context, Product and Manufacturing Information (PMI) modules available in modern CAD systems are already popular among engineers and designers so they are natural vehicles to interact with annotations [15].

\subsection{Annotation Visualization}

From an interaction standpoint, an ever increasing number of annotations can quickly result in a cluttered model, which often creates confusion and a feeling of information overload in the user. When too much data (or when data is not well organized) is displayed on a too small area, the value of information diminishes [31].

Previous studies on visual clutter have focused on algorithms for annotation styles [32], layouts to prevent occlusion [33], and the automatic arrangement of information [34]. However, none of the current model-based standards provides guidelines to reduce visual clutter (although they do recommend the use of groups to simplify interaction), and thus, no implementations are available in current PMI modules. Advanced filtering and interactive navigation based on the model's features have been proposed [15] as alternatives. These methods are generally faster as they do not rely on the user to create the groups and distribute the annotations within these groups.

\subsection{User Motivation}

Most annotation and knowledge representation techniques have proven to be valuable, but the majority do not find acceptance in industry, as designers are reluctant to spend time annotating their designs [35]. One reason is that the designer that has to implement the annotations has no further use of them. Why should the designer do something that is only beneficial for people that come after her? In many cases, incentives are missing.

Convincing users to use annotations can be a challenge, especially if the argument focuses exclusively on the collaborative aspect of helping other users. Even if the designer is forced to annotate her work, it is unclear that she will create quality annotations. On the other hand, just as computer programmers comment their source code to document algorithms, designers also need proper documentation to remember all model changes. Therefore, automatic tools that kept a historical record of annotation information could motivate and incentivize designers. After all, historical annotation information may not just be valuable for future users of a model, but also for the original creators. 


\subsection{Summary of Approaches}

A summary of the most representative 3D annotation approaches and how they implement the challenges presented are shown in Table 2.

Table 2. Summary of 3D annotation approaches

\begin{tabular}{lllll}
\hline Approach & storage & representation & interface & visualization \\
\hline Multiple Viewpoint [17] & Stand-off & Structured & Add-on & Not specified \\
LIMMA [23] & Stand-off & $\begin{array}{l}\text { Freestyle and } \\
\text { Structured }\end{array}$ & Add-on & Not specified \\
MATRICS [36] & Not specified & Freestyle & Knowledge-Based & Not specified \\
Space Pen [37] & Inline & Freestyle & Pen & Not specified \\
3DAF [38] & Stand-off & Structured & Knowledge- Based & Not specified \\
Web-standards [39] & Inline and & Structured & Knowledge-Based & Not specified \\
& Stand-off & & & Not specified \\
\hline
\end{tabular}

\section{Discussion}

The use of annotations as tools to incorporate product information within CAD models has proven to be a viable option for collaborative product development activities, partly because of the standardization of practices and the popularization of the Model-Based Enterprise paradigm. However, important challenges appear if the role of annotations is extended to communicate design information. Although some studies suggest that annotations may serve this purpose effectively, proper mechanisms must be put in place. Specifically, the problems of visual clutter, effective interfaces, and the automatic processing of freestyle text are challenges that will likely be addressed by upcoming versions of standards and PMI modules. User motivation challenges require integrated approaches that simplify annotation processes and incentivize users to document their designs.

In this review paper, we have examined the background, approaches, and issues in model annotation technology, as well as the impact of these mechanisms on design communication. This review is a crucial step to identify the aspects that are relevant to collaborative design activities. Selected technical literature has been analyzed to determine the aspects that must be considered in practical implementations.

The existing gap between engineering design and communication technologies is gradually being reduced as access to information becomes easier and new frameworks for product development become more distributed and collaborative. The use of model annotations to represent, capture and reuse design information is a promising subject, but mechanisms for indexing, searching, processing, and retrieving this information are needed. Additionally, commercial CAD manufacturers must provide tools that facilitate knowledge capture, reuse, and integration. It can be expected that collaborative design processes will be substantially affected by new advancements in annotation technologies. 


\section{References}

1. Katzenbach, J.R., Smith, D.K.: The Discipline of Teams. Harvard Business Review 71(2), 111-120 (2005)

2. Campion, M.A., Medsker, G.J., Higgs, A.C.: Relations between Work Group Characteristics and Effectiveness: Implications for Designing Effective Work Groups. Personnel Psychology 46, 823-850 (1993)

3. Chudoba, K.M., Wynn, E., Lu, M., Watson-Manheim, M.B.: How Virtual Are We? Measuring Virtuality and Understanding its Impact in a Global Organization. Information Systems Journal 15, 279-306 (2005)

4. Lahti, H., Seitamaa-Hakkarainen, P., Hakkarainen, K.: Collaboration Patterns in Computer Supported Collaborative Designing. Design Studies 25, 351-371 (2004)

5. Chang, K.H., Silva, J., Bryant, I.: Concurrent Design and Manufacturing for Mechanical Systems. Concurrent Engineering 7, 290-308 (1999)

6. Jackson, C., Buxton, M.: The Design Reuse Benchmark Report: Seizing the Opportunity to Shorten Product Development. Aberdeen Group, Boston (2007)

7. Lang, SYT., Dickinson, J., Buchal, R.O.: Cognitive Factors in Distributed Design. Computers in Industry 48, 89-98 (2002)

8. Alemanni, M., Destefanis, F., Vezzetti, E.: Model-Based Definition Design in the Product Lifecycle Management Scenario. International Journal of Advanced Manufacturing Technology, 52(1-4) , 1-14 (2011)

9. ASME: ASME Y14.41-2012 Digital Product Definition Data Practices. The American Society of Mechanical Engineers, New York (2012)

10.ISO: ISO 16792:2006 Technical Product Documentation - Digital Product Definition Data Practices. Organisation Internationale de Normalisation, Genève, Suisse (2006)

11.Bracewell, R.H., Wallace, K.M.: A Tool for Capturing Design Rationale. In: 14th International Conference on Engineering Design, Design Society,Stockholm,Sweden (2003)

12.Boujut, J.F., Dugdale, J.: Design of a 3D Annotation Tool for Supporting Evaluation Activities in Engineering Design. Cooperative Systems Design, COOP 6, 1-8 (2006)

13.Alducin-Quintero, G., Rojo, A., Plata, F., Hernández, A., Contero, M.: 3D Model Annotation as a Tool for Improving Design Intent Communication: A Case Study on its Impact in the Engineering Change Process. In: ASME International Design Engineering Technical Conferences \& Computers and Information in Engineering Conference. Chicago, Illinois (2012).

14.Sandberg, S., Näsström, M.: A Proposed Method to Preserve Knowledge and Information by Use of Knowledge Enabled Engineering. In: ASME International Design Engineering Technical Conferences \& Computers and Information in Engineering Conference, Las Vegas, Nevada (2007)

15.Dorribo-Camba, J., Alducin-Quintero, G, Perona, P., Contero, M.: Enhancing Model Reuse through 3D Annotations: A Theoretical Proposal for an Annotation-Centered Design Intent and Design Rationale Communication. In: ASME International Mechanical Engineering Congress \& Exposition, San Diego, California (2013)

16.Ding, L., Ball, A., Patel, M., Matthews, J., Mullineux, G.: Strategies for the Collaborative Use of CAD Product Models. In: 17th International Conference on Engineering Design, vol. 8, 123-134 (2009)

17.Davies D., McMahon C.A.: Multiple Viewpoint Design Modelling through Semantic Markup. In: ASME International Design Engineering Technical Conferences and Computers and Information in Engineering Conference, Philadelphia, PA. vol. 3, pp. 561-571 (2006)

18.Pena-Mora, F., Sriram, D., Logcher, R.: SHARED-DRIMS: SHARED Design Recommendation-Intent Management System. Enabling Technologies: Infrastructure for Collaborative Enterprises, 213-221 (1993) 